
online version of the article only. Distribution for non-commercial purposes only.

\title{
Mussel Shell Impaction in the Esophagus
}

\author{
Sunmin Kim Hyung Hun Kim Gook Hwan Jang Jun Young Song \\ Department of Internal Medicine, Kosin University College of Medicine, Busan, Korea
}

\section{Key Words}

Mussels · Mussel shell impaction · Esophagus · Endoscopy

\begin{abstract}
Mussels are commonly used in cooking around the world. The mussel shell breaks more easily than other shells, and the edge of the broken mussel shell is sharp. Impaction can ultimately cause erosion, perforation and fistula. Aside from these complications, the pain can be very intense. Therefore, it is essential to verify and remove the shell as soon as possible. In this report we describe the process of diagnosing and treating mussel shell impaction in the esophagus. Physicians can overlook this unusual foreign body impaction due to lack of experience. When physicians encounter a patient with severe chest pain after a meal with mussels, mussel shell impaction should be considered when diagnosing and treating the patient.
\end{abstract}

\section{Introduction}

Foreign body ingestion is a common presenting problem in the emergency department. While the vast majority of ingested foreign bodies traverse the gastrointestinal tract with nil adverse sequelae $[1,2]$, some require early endoscopic removal due to their corrosive nature [3], potential for alimentary tract perforation [4] or physical size [4]. In adults, etiologic factors associated with foreign body ingestion include poor visual acuity, alcohol and drug addiction, dental prostheses and psychiatric disease [5]. The management of ingested foreign objects depends on the nature of the ingested object, its location at presentation and other patient factors. While the vast majority of foreign bodies that reach the stomach will traverse the alimentary tract spontaneously $[1,2,6]$, requiring little more than observation in an outpatient setting, a few require targeted endoscopic or surgical removal to prevent complications of impaction, obstruction, or perforation leading to abscess formation, fistulae or generalized peritonitis. Here, we report the case of a 28-year-old man who accidentally 
Kim et al.: Mussel Shell Impaction in the Esophagus

swallowed a piece of mussel shell, which was extracted via endoscopy without any complications.

\section{Case Report}

A 28-year-old man presented to the emergency room for persistent deep thoracic pain. He reported that the pain was so intense that he could not even sit down by himself. The patient reported the onset of acute sharp pain after a meal of seafood stew and rice $1 \mathrm{~h}$ before admission. Notably, the patient drank the broth of the stew. There were no abnormalities on electrocardiogram. Plain neck films demonstrated an approximately 2-cm-sized radiopaque linear flat object in front of the 6th and 7th cervical vertebrae, which was only visible on lateral projection (fig. 1). Cervicothoracic computed tomography revealed a highly attenuated thin linear foreign body impacted in the esophagus at the level of the 6th to 7th cervical vertebrae (fig. 2). Urgent esophagoscopy was immediately performed, and a large piece of a mussel shell was found. The shell appeared nonobstructive but was impacted within the mucosal surface of the mid-esophagus (fig. 3). It was successfully extracted through a bag to avoid damaging the esophagus. The removed shell was about a 2 -cm-sized triangle with sharp edges (fig. 4). The pain immediately resolved after removal of the shell. A follow-up endoscopy performed the next day showed that the esophageal mucosa was intact.

\section{Discussion}

As a group, esophageal foreign bodies require early intervention (within $24 \mathrm{~h}$ ) because of their potential to cause respiratory complications, esophageal erosions or, in the most extreme instances, aortoesophageal fistula [7]. Ingested button batteries that become lodged in the esophagus require still greater expedition of removal given the risk of corrosive tissue damage [3]. Swallowed linear foreign bodies of $>6-10 \mathrm{~cm}$ length, such as toothbrushes, present a unique problem in that, given their length, they are unable to negotiate the curvature of the duodenum with its fixed retroperitoneal attachment [8]. Recent recognition of the dangers posed by multiple ingested magnets, due to pressure necrosis of the bowel wall caught between adjacent magnets, emphasizes the importance of vigilance and consideration of early endoscopic removal before small bowel transit [9]. Likewise, sharp foreign bodies require endoscopic removal due to the increased risk of gastrointestinal perforation (15-35\%) [2].

The word 'mussel' is most frequently used to reference the edible bivalves of the marine family Mytilidae, most of which live on exposed shores in the intertidal zone, attached to a firm substrate by their strong byssal threads. In most marine mussels, the shell is longer than it is wide, giving it an asymmetrical wedge shape. The external color of the shell is often dark blue, blackish or brown, while the interior is silvery and somewhat nacreous. Humans have consumed mussels as food for thousands of years and continue to do so. About 17 species are edible, of which the most commonly eaten are Mytilus edulis, M. galloprovincialis, M. trossellus and Perna canaliculus. One of the most famous foods that contain mussels is Italian seafood pasta, in which mussels are often mixed with other seafood or eaten with pasta. This report demonstrates that a serious problem with mussels is that their shell can be easily broken. Moreover, the edges of the broken shells are very sharp (fig. 4). If a person swallows a broken piece of mussel shell, it can easily impact the wall of the esophagus. Consequently, the patient invariantly presents with severe pain. In fact, a doctor in the 
emergency room suspected a cardiogenic problem when he met the patient due to the patient's severe chest pain.

As mussel shell impaction can create esophageal erosion, perforation and aortoesophageal fistula, it is necessary to identify it and remove it as soon as possible. Fortunately, the mussel shell is radiopaque, so it can be detected by radiologic examination. Neck and chest plain film can also be used to locate the mussel shell. Interestingly, only the lateral view will reveal the shell. In both the case that we present and another report, the shell could not be visualized in the posteroanterior or anteroposterior view [10]. In contrast, a short-neck clam impaction exhibited a radiopaque silhouette in both the posteroanterior and the lateral view [11]. This phenomenon appears to be due to the characteristics and the position of the mussel shell. The mussel shell is thinner compared with the shells of other shellfishes; it is located parallel to the coronal section of the body (fig. 3), so it is naturally more radiopaque in the lateral view. Thus, careful observation of lateral view images is necessary when we suspect mussel shell impaction. Cervicothoracic computed tomography helps us accurately identify the shape and location of the lesion. All three cases, including ours, showed that the shell was located in the narrowing of the aortic arch $[10,11]$. The foreign body usually lies close to one of the three esophageal anatomical constrictions: the cricopharyngeal ring, the narrowing of the aortic arch or the esophagogastric junction [12,13].

Therefore, it is essential to verify and remove the shell as soon as possible when mussel shell impaction is suspected. A careful history of food intake will provide the most important information that will raise clinical suspicion for possible mussel shell impaction. Then, lateral views of the neck and chest film should be obtained and carefully examined. Computed tomography can confirm the diagnosis and guide endoscopic removal. When physicians encounter a patient with severe chest pain after a meal with mussels, mussel shell impaction should be considered when diagnosing and treating the patient.

\section{References}

1 Antoniou D, Christopoulos-Geroulanos G: Management of foreign body ingestion and food bolus impaction in children: a retrospective analysis of 675 cases. Turk J Pediatr 2011;53:381-387.

-2 Kay M, Wyllie R: Pediatric foreign bodies and their management. Curr Gastroenterol Rep 2005;7:212-218.

3 Jarugula R, Dorofaeff T: Oesophageal button battery injuries: think again. Emerg Med Australas 2011;23: 220-223.

4 Henderson CT, Engel J, Schlesinger P: Foreign body ingestion: review and suggested guidelines for management. Endoscopy 1987;19:68-71.

5 Mosca S, Manes G, Martino R, Amitrano L, Bottino V, Bove A, Camera A, De Nucci C, Di Costanzo G, Guardascione M, Lampasi F, Picascia S, Picciotto FP, Riccio E, Rocco VP, Uomo G, Balzano A: Endoscopic management of foreign bodies in the upper gastrointestinal tract: report on a series of 414 adult patients. Endoscopy 2001;33:692-696.

6 Chiu YH, Hou SK, Chen SC, How CK, Lam C, Kao WF, Yen DH, Huang MS: Diagnosis and endoscopic management of upper gastrointestinal foreign bodies. Am J Med Sci 2012;343:192-195.

7 Lai H, Ge D, Zheng YJ, Li J, Wang C: Surgical management of aortoesophageal fistula caused by foreign bodies. Eur J Cardiothorac Surg 2011;40:13-16.

$>8$ Eisen GM, Baron TH, Dominitz JA, Faigel DO, Goldstein JL, Johanson JF, Mallery JS, Raddawi HM, Vargo JJ 2nd, Waring JP, Fanelli RD, Wheeler-Harbough J: Guideline for the management of ingested foreign bodies. Gastrointest Endosc 2002;55:802-806.

9 Naji H, Isacson D, Svensson JF, Wester T: Bowel injuries caused by ingestion of multiple magnets in children: a growing hazard. Pediatr Surg Int 2012;28:367-374.

10 Coulier B, Vander Elst B, Van Hoof M: Oesophageal impaction of a mussel shell. JBR-BTR 2008;91:61.

11 Pelucchi S, Bianchini C, Ciorba A, Pastore A: Unusual foreign body in the upper cervical oesophagus: case report. Acta Otorhinolaryngol Ital 2007;27:38-40.

12 von Rahden BH, Feith M, Dittler HJ, Stein HJ: Cervical esophageal perforation with severe mediastinitis due to an impacted dental prosthesis. Dis Esophagus 2002;15:340-344.

13 Chadha SK, Gopalakrishnan S, Gopinath N: An unusual sharp foreign body in the esophagus and its removal. Otolaryngol Head Neck Surg 2003;128:766-768. 
Kim et al.: Mussel Shell Impaction in the Esophagus

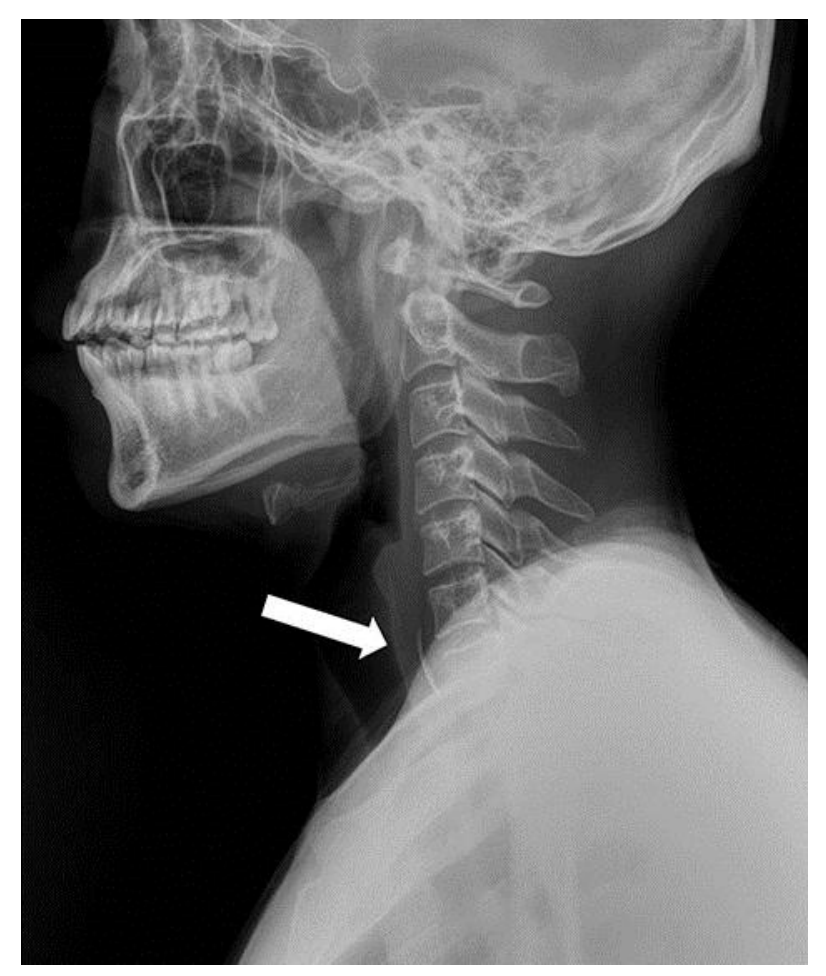

Fig. 1. In a plain neck lateral film, an approximately 2-cm-sized radiopaque linear flat foreign body (arrow) at the level of the 6th and 7th cervical vertebrae could be clearly identified. No bony or soft tissue abnormalities were demonstrated.

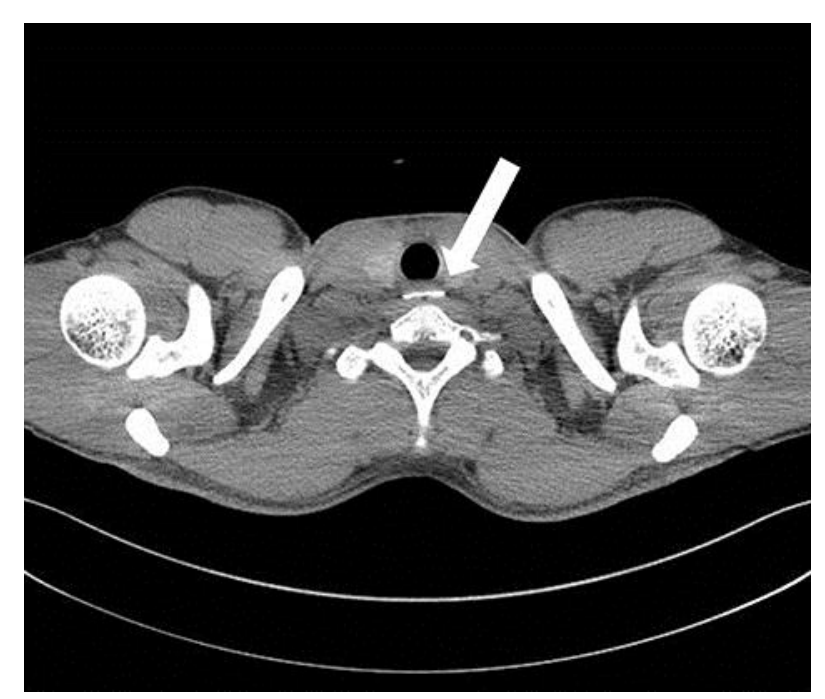

Fig. 2. Cervicothoracic computed tomography showed a highly attenuated, bone like, thin linear material impacted inside the cervical esophagus at the level of 6 th to 7 th cervical vertebrae. There were no definite complications caused by foreign body impaction, such as perforation and abscess. 
Kim et al.: Mussel Shell Impaction in the Esophagus

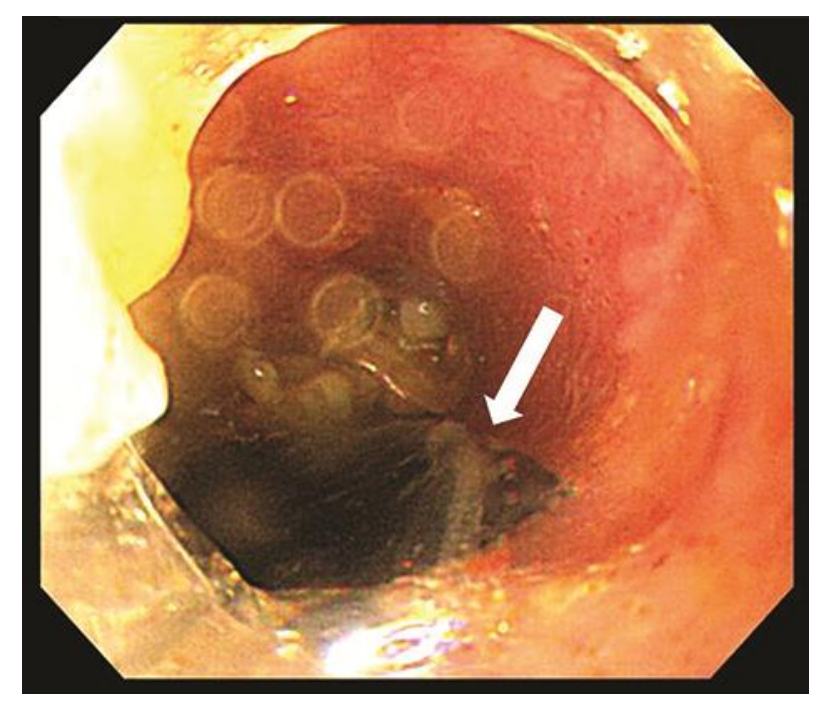

Fig. 3. Esophagoscopy demonstrated a blackish fragment impacted in the esophagus. The piece of the shell was impacted tightly and did not move easily when the endoscopist touched it with a forceps. We used a bag when extracting the shell to avoid damaging the esophagus and upper esophageal sphincter.

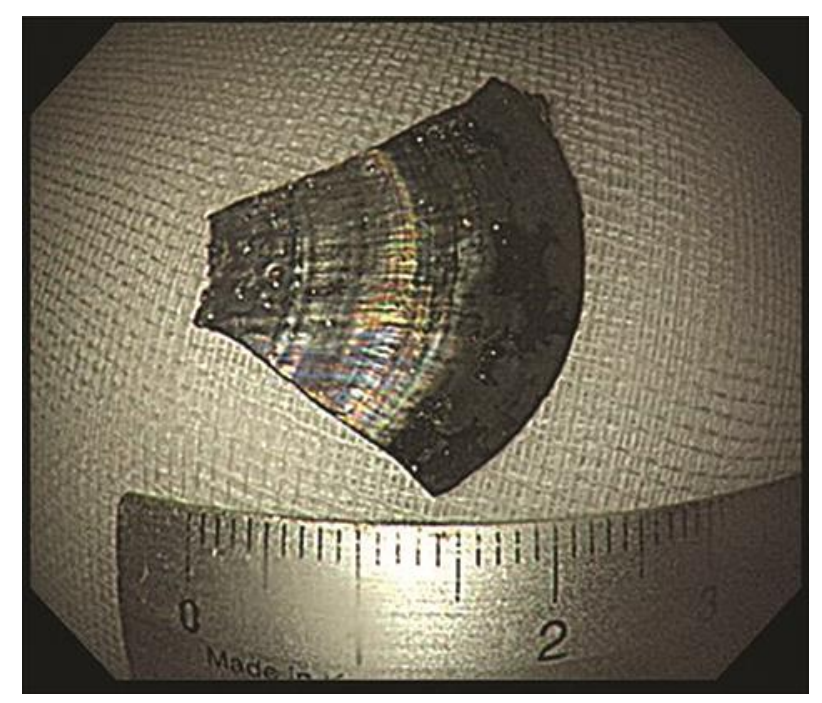

Fig. 4. The extracted piece of mussel shell had a triangular shape with very sharp edges. The shell was about $2 \mathrm{~cm}$ in size. These sharp edges may cause serious pain and tight impaction in the esophagus. 